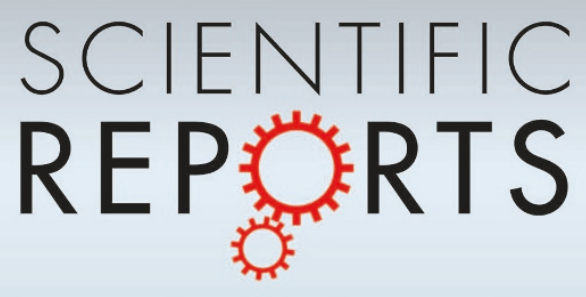

OPEN

SUBJECT AREAS:

PHASE TRANSITIONS

AND CRITICAL

PHENOMENA

THERMODYNAMICS

STRUCTURE OF SOLIDS AND

LIQUIDS

CHEMICAL BONDING

Received

11 April 2013

Accepted

11 July 2013

Published

5 August 2013

Correspondence and requests for materials should be addressed to V.L.S. (vladimir. solozhenko@univparis 13.fr)

\section{Equilibrium p-T Phase Diagram of Boron: Experimental Study and Thermodynamic Analysis}

\author{
Vladimir L. Solozhenko' \& Oleksandr O. Kurakevych ${ }^{2}$
}

'LSPM-CNRS, Université Paris Nord, 93430 Villetaneuse, France, ${ }^{2}$ IMPMC, Université P\&M Curie, 75005 Paris, France.

Solid-state phase transformations and melting of high-purity crystalline boron have been in situ and ex situ studied at pressures to $20 \mathrm{GPa}$ in the $1500-2500 \mathrm{~K}$ temperature range where diffusion processes become fast and lead to formation of thermodynamically stable phases. The equilibrium phase diagram of boron has been constructed based on thermodynamic analysis of experimental and literature data. The high-temperature part of the diagram contains $p$ - $T$ domains of thermodynamic stability of rhombohedral $\beta-B_{106}$, orthorhombic $\gamma-B_{28}$, pseudo-cubic (tetragonal) $t^{\prime}-B_{52}$, and liquid boron (L). The positions of two triple points have been experimentally estimated, i.e. $\beta-\mathrm{t}^{\prime}-\mathrm{L}$ at $\sim 8.0 \mathrm{GPa}$ and $\sim 2490 \mathrm{~K}$; and $\beta-\gamma-\mathrm{t}^{\prime}$ at $\sim 9.6 \mathrm{GPa}$ and $\sim 2230 \mathrm{~K}$. Finally, the proposed phase diagram explains all thermodynamic aspects of boron allotropy and significantly improves our understanding of the fifth element.

T he phase diagram of boron and thermodynamic stability of boron allotropes remain of fundamental interest in condensed matter physics and chemistry for a very long time - since the first characterization of pure element's allotropes in $1950 \mathrm{~s}^{1-3}$. Later the existence of multiple boron modifications has been reported (see recent reviews ${ }^{2,3}$ ) but some of them never have been reproduced, e.g. $\beta^{\prime}$ - and $\beta^{\prime \prime}-\mathrm{B}^{4}, \mathrm{t}-\mathrm{B}_{50}{ }^{5}$, "HP form" of Wentorf ${ }^{6}$, "HPHT form" of $\mathrm{t}-\mathrm{B}_{192}{ }^{7}$, etc. At present time only five allotropes are generally accepted: rhombohedral $\alpha-\mathrm{B}_{12}$ $(\alpha \text {-phase })^{8}$ and $\beta-B_{106}(\beta \text {-phase })^{9}$, orthorhombic $\gamma-B_{28}(\gamma \text {-phase })^{10}$, tetragonal $t-B_{192}{ }^{11}$ and $t-B_{52}(t-p h a s e$ in this paper $)^{5,12-14} \cdot t-B_{52}$ has been proved to exist only very recently and its crystal structure has not been unambiguously established so far. Two more phases have been predicted using $a b$ initio structural evolution algorithm ${ }^{15}$, i.e. orthorhombic $0-B_{52}{ }^{16}$, closely related to $t-B_{52}$, and metallic boron with $\alpha$-Ga crystal structure ${ }^{10}$. The interest to the high-pressure behavior of boron has recently raised due to the discovery of boron superconductivity at high pressure $^{17,18}$; unusual partially ionic character of some B-B bondings in $\gamma$-boron ${ }^{10}$; high-pressure synthesis of novel boron-rich compounds ${ }^{19-26}$ that are refractory and chemically stable ${ }^{27-29}$, superhard ${ }^{30-32}$, and even could have metallic conductivity ${ }^{33}$; unusual pressure-induced behavior of boron-containing icosahedral ${ }^{14,35}$ and layered ${ }^{36-39}$ structures; and prediction of the nonmetal-metal phase transition in boron at a pressure above $89 \mathrm{GPa}^{10,40}$.

The stability of boron allotropes has been intensively investigated during past years using ab initio calculations. It has been predicted that at ambient conditions $\alpha-B_{12}$ and $\beta-B_{106}$ have similar static energies, but disordered $\beta-B_{106}$ is more stable at ambient pressure, due to its lower zero-point vibrational energy ${ }^{41}$. At pressures above $2 \mathrm{GPa}$, denser $\alpha-\mathrm{B}_{12}$ should be more stable ${ }^{10}$. The ab initio analysis of stability of boron structures ${ }^{10}$ showed that at pressures above $20 \mathrm{GPa}$ the $\alpha$-phase loses its stability and another phase, orthorhombic $\gamma-\mathrm{B}_{28}$ (confirmed experimentally ${ }^{10}$ ), becomes stable. Finally, above $89 \mathrm{GPa}$ transition of semiconductive $\gamma$-phase into metallic one should occur ${ }^{10}$. However, this pressure domain has not been explored experimentally at high temperatures (to overcome a kinetic barrier), and the latter allotrope remains to be discovered. Thus, numerous theoretical predictions require rigorous experimental studies.

The first attempt to analyze the high-pressure phase equilibria in boron was made in $2007^{42}$ based on ab initio calculations for $\alpha-B_{12}$ and $\beta-B_{106}$ phases and some ambient pressure experimental data. However, the result contradicts the experimental data on boron melting under pressure ${ }^{43,44}$, i.e. $\mathrm{d} T_{\mathrm{m}} / \mathrm{d} p$ is overestimated by a factor of 2 (see Fig. 1c). Moreover, the reported $\alpha \leftrightarrows \beta$ equilibrium temperature $\left(T_{\beta / \alpha}\right)$ at ambient pressure is lower by $\sim 200 \mathrm{~K}$ as compared with the maximal temperature of $\beta$-to- $\alpha$ recrystallization in the presence of Pt melt ${ }^{45}$.

The first $p$ - $T$ phase diagram of boron was proposed only in 2009 by Oganov et al. ${ }^{10}$ and contains 5 allotropes (four experimentally confirmed forms $\alpha, \beta, \gamma, \mathrm{t}-\mathrm{B}_{192}$, and hypothetical metallic one) and liquid boron. This diagram combined more extended ab initio and experimental data on structural stability and phase relationships, but still some points remained unclear. Though overall correct, this phase diagram contained an uncertainty 


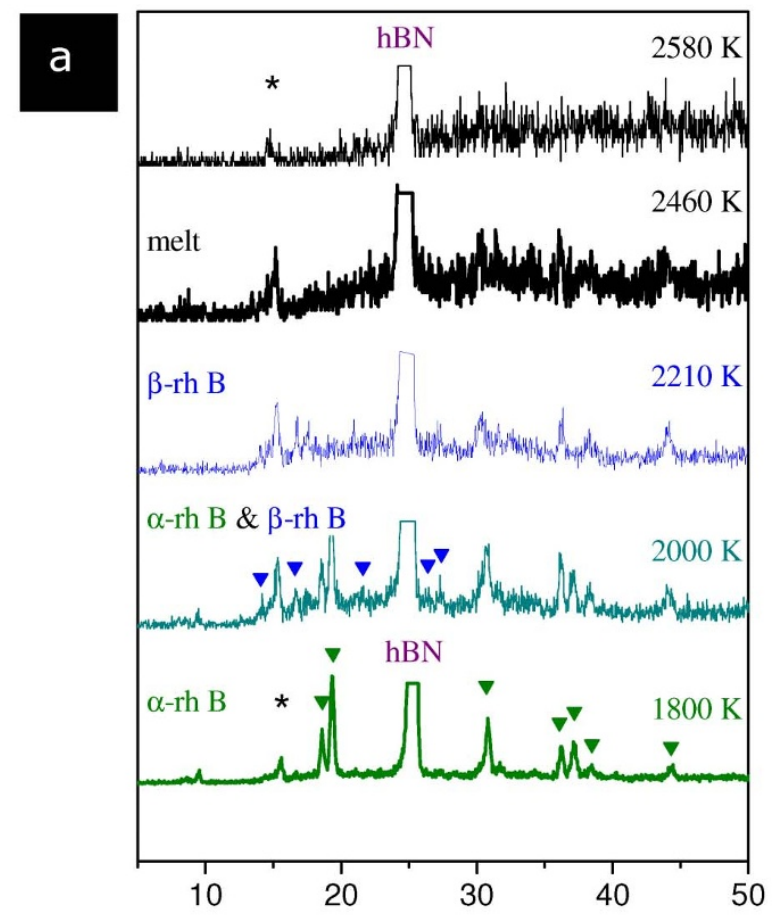

\section{$E, \mathrm{keV}$}

\section{b}
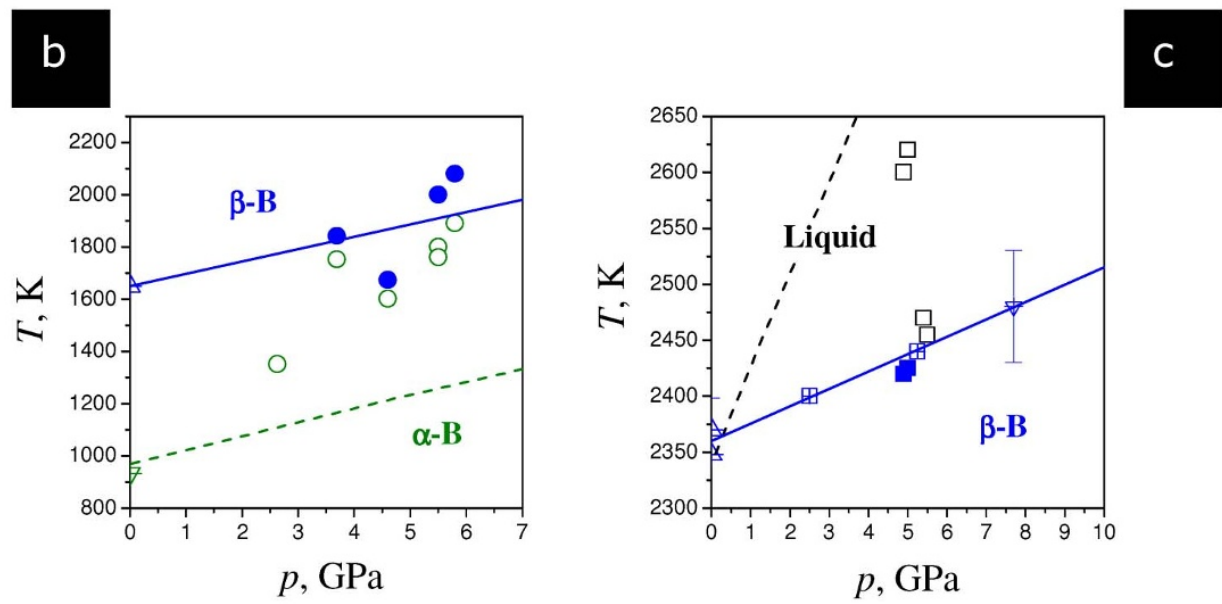

Figure $1 \mid$ (a) Sequence of synchrotron energy-dispersive X-ray diffraction patterns taken in situ in the course of stepwise heating of $\alpha$-boron at 5.5 GPa $(E \cdot d=78.07 \mathrm{eV} \mathrm{\AA})$. Asterisk $\left(^{*}\right)$ indicates the position of the escape line of boron nitride (hBN, capsule material). (b) Experimental data on $\alpha$-to- $\beta$ transformation in boron. Open and solid circles represent the $\alpha$ - and $\beta$-phases observed during our in situ experiments. The up triangle corresponds to the beginning of the $\alpha$-to- $\beta$ solid-state transformation ${ }^{4}$, while the down triangle is the onset of recrystallization of $\alpha$ - $B_{12}$ from $\beta$-B $B_{106}$ in the presence of Pt melt $t^{50}$. The dashed line corresponds to the equilibrium between $\alpha-B_{12}$ and $\beta-B_{106}$ that has been calculated in Ref.42. (c) Experimental data on boron melting. Solid and open squares represent the crystalline and liquid boron observed during our in situ experiments. The crossed squares correspond to the beginning of melting. The open triangles show the literature data: $(2350 \mathrm{~K} \text {, ambient pressure })^{60},(2370 \mathrm{~K} \text {, ambient pressure })^{61}$ and $(2480 \mathrm{~K}, 7.7 \mathrm{GPa})^{43}$. The dashed line represents the melting curve calculated in Ref.42.

related to the stability field of the tetragonal boron phase, which at that time was thought to be "HPHT t- $\mathrm{B}_{192}{ }^{\text {"7 }}$. A second point requiring elaboration is that the equilibrium line between $\alpha$ - and $\beta$-boron was an estimate, rather than direct measurement (which would be complicated by kinetics) or calculation (which would be complicated by disorder in $\beta$-boron). Recrystallization of $\alpha-B_{12}$ from $\beta-B_{106}$ was experimentally observed at much higher temperatures at both ambient (from melts containing $\mathrm{Pt}^{45}$ ) and high (during solid-state transformation $^{12}$ ) pressures. Finally, $t-B_{52}$ has been recently obtained at ambient ${ }^{12}$ and high ${ }^{12-14}$ pressures, and even recovered as a single phase $\mathrm{e}^{12,14}$. Although this tetragonal allotrope has been interpreted as a metastable one as compared to mysterious "HPHT t- $\mathrm{B}_{192}$ ", our recent results showed that the latter has a crystal structure related in many aspects to $t-B_{52}$ phase, rather than to $t-B_{192}{ }^{46}$.

Very recently pseudo-cubic $\mathrm{t}^{\prime}-\mathrm{B}_{52}$ of the $\mathrm{t}-\mathrm{B}_{52}$ structural family has been discovered ${ }^{46}$. It was recovered after experiments at $20 \mathrm{GPa}$ and $2500 \mathrm{~K}$, the highest temperature reported so far for formation of a tetragonal phase. Contrary to common low-density t- $\mathrm{B}_{52}$ phase(s) and related compounds, pseudo-cubic allotrope is quite dense, very close to $\gamma-B_{28}$. This phase seems to be a good candidate for a HPHT 
allotrope, instead of strongly distorted "t-B $B_{192}$ structure". At such high temperature the diffusion processes are quite intense even at such high pressure as $20 \mathrm{GPa}$. The observation of reversible transformation in boron at HPHT conditions would be a strong support to equilibrium phase diagram.

In the present work we have studied the high-temperature part of boron phase diagram using in situ and recovery high-pressure experiments (to $20 \mathrm{GPa}$ and $2500 \mathrm{~K}$ ), as well as thermodynamic analysis. The established equilibria between boron allotropes and liquid phase are self-consistent from the point of view of classical thermodynamics and adequately describe the experimental data obtained by independent research groups.

\section{Results}

Phase transformation of $\boldsymbol{\alpha}-\mathbf{B}_{12}$ into $\boldsymbol{\beta}-\mathbf{B}_{106}$. Our in situ studies of $\alpha$-boron in the 2-6 GPa pressure range using MAX80 multianvil system and energy-dispersive synchrotron X-ray powder diffraction at HASYLAB-DESY ${ }^{47}$ have shown that the phase transformation of $\alpha-B_{12}$ into $\beta-B_{106}$ occurs at temperatures above $1600 \mathrm{~K}$ (Fig. 1a \& b). It passes noticeably higher the stability line predicted in Ref. 42 and shows strongly non-monotone temperature dependence in the 1600-1800 $\mathrm{K}$ range. At higher temperatures, 2400$2500 \mathrm{~K}$, all lines of $\beta-\mathrm{B}_{106}$ disappear due to the melting (Fig. 1a \& c).

Two opinions are know in the literature on the thermodynamical stability of $\alpha-B_{12}$, i.e. (i) at low and moderate pressures it has its own domain in the $p-T$ phase diagram ${ }^{42}$, (ii) below $8 \mathrm{GPa} \beta-\mathrm{B}_{106}$ phase is stable allover the $p-T$ region up to the melting temperature ${ }^{43,48}$. Since the $\alpha \leftrightarrows \beta$ equilibrium temperature obtained from $a b$ initio calculations $^{42,49}$ is lower by $\sim 800 \mathrm{~K}$ than the onset temperature of the $\alpha$-to- $\beta$ transformation (Fig. 1b), one cannot make a clear conclusion whether the crystallization of $\alpha$-boron under these conditions is equilibrium or kinetically limited.

Very recently an attempt to study the vicinity of the $\alpha-\beta-\gamma$ triple point has been made by Parakhonskiy et al. ${ }^{50}$. Although the data on the growth of single-crystal $\alpha-B_{12}$ in metal systems is interesting by itself, the authors' interpretation of the results in terms of equilibrium thermodynamics is quite contradictory. From one side, at temperatures of $1400-1600 \mathrm{~K}$ the numerous metastable phases crystallize $^{12,51}$, especially, on the timescale of Ref.50. Moreover, the extrapolation of the $\alpha \leftrightarrows \beta$ equilibrium line reported in Ref.50 down to $1 \mathrm{MPa}$ contradicts the crystallization of $\alpha-\mathrm{B}_{12}$ from $\mathrm{Pt}$ melt at higher (at least, $200 \mathrm{~K}$ higher than in Ref.50) temperatures ${ }^{45}$, i.e. in the domain of stability of $\beta-B_{106}$ phase. At such low temperatures and for such rigid structures the formation enthalpy of $\alpha-B_{12}$ from $\beta-B_{106}$ $\left(\Delta H_{\alpha / \beta}\right)$ is more important for the estimation of thermodynamic stability of a phase than the fact of single crystal growth, which can occur outside the domain of thermodynamic stability, especially, in the case of covalent cage structures (e.g. Si clathrates ${ }^{52}$ ). Although Parakhonskiy et al. ${ }^{50}$ believe that their approach is similar to the classical study of the graphite $\leftrightarrows$ diamond equilibrium, the close (in contrast to graphite/diamond) structural relationships between boron allotropes ${ }^{4,10}$ can result in the metastable crystal growth of boron according to the Ostwald rule of stages. Moreover, the ability of boron to host transition metals ( $\mathrm{Pt}$, in particular) in the structure ${ }^{53}$ was completely neglected by Parakhonskiy et al. ${ }^{50}$, while it can influence the mutual stability of various structures and the crystallization order (for example, metastable nitrogen-doped $t-B_{52}$ was observed in the $\mathrm{B}-\mathrm{BN}$ system already at $5 \mathrm{GPa}^{20,22,29}$ ).

To perform thermodynamic analysis of relative stabilities of $\alpha-B_{12}$ and $\beta-B_{106}$, we fitted experimental heat capacities to the Holzapfel equation $^{54,55}$ (Fig. 2a, Tab. 128,56-58). The estimates for different contributions into total Gibbs energy $\Delta G_{\alpha / \beta}$ (solid curves) are presented in Fig. 2b as dashed curves: (1) thermal due to the difference in heat capacities, and (2) configurational due to non-zero configurational entropy of $\beta-B_{106}{ }^{1,49}$. Taking into account only thermal part would lead to astonishing result: the $\alpha-B_{12}$ phase should be stable either at high temperature or in the whole temperature range with very narrow $(500-750 \mathrm{~K})$ stability domain of $\beta-\mathrm{B}_{106}$. This fact contradicts to all known experimental data on phase relationships between these two boron allotropes and cannot be overcome by variation of $\Delta H_{\alpha / \beta}$ value. Only including the configurational entropy (the lower estimate based on structural data from Ref.59 is $\left.\sim 0.5 R \mathrm{Jmol}^{-1} \mathrm{~K}^{-1}\right)^{49}$ allows us to obtain reasonable $\Delta G_{\alpha / \beta}$ dependencies (Fig. $2 \mathrm{~b}$, solid curves). The impact of configurational entropy on thermodynamic properties is remarkable only in the case of $\beta-\mathrm{B}_{106}$ (standard state): $\alpha-B_{12}$ and $\gamma-B_{28}$ have zero configurational entropy, while that of pseudo-cubic $\mathrm{t}^{\prime}-\mathrm{B}_{52}$ is "hidden" in the corresponding $\Delta S_{\mathrm{X} / \mathrm{t}}$ ' (quasi-constant at high temperatures) values describing the $\mathrm{X} \leftrightarrows \mathrm{t}$ ' equilibria.

Now, for the $\alpha \leftrightarrows \beta$ "equilibrium" line reported in Ref.50 the $\Delta H_{\alpha / \beta}$ value should be about $-4.5 \mathrm{~kJ} / \mathrm{mol}$ (Fig. 2b) but even this "lower estimate" for $\Delta H_{\alpha / \beta}$ seems to be too high as compared to both the results of $a b$ initio calculations $s^{10,49}$ and experimental value of $\Delta H_{\text {amorphous } / \beta}{ }^{57}$. Thus, the experimental estimation of $\Delta H_{\alpha / \beta}$ (as well as $S_{\text {config. }}$ of $\beta-B_{106}$ ) seems to be the crucial point for establishing topology of the boron phase diagram in the vicinity of the $\alpha-\beta-\gamma$ triple point and at lower temperatures. So far, the $\alpha \leftrightarrows \beta$ and $\alpha \leftrightarrows \gamma$ equilibrium lines reported in Ref.50 may be only considered as overestimated upper boundaries of the thermodynamic stability domain of $\alpha-B_{12}$. Moreover, the most recent experimental ${ }^{1}$ and theoretical ${ }^{10}$ studies clearly indicate that the $\alpha \leftrightarrows \beta$ equilibrium line crosses the pressure axis, in contrast to Ref.50.

Fig. $2 \mathrm{c}$ shows the tentative low-temperature part of the boron phase diagram. The $\alpha \leftrightarrows \beta$ equilibrium line intersects the pressure axis, similar to previous simulations ${ }^{1,10}$. The slope $(\mathrm{d} p / \mathrm{d} T)_{\alpha-\beta}=$ $\Delta S_{\alpha-\beta} / \Delta V_{\alpha-\beta}$ is positive and has a non-zero value at $0 \mathrm{~K}$ due to the configurational enthropy of $\beta-\mathrm{B}_{106}$. Our lowest estimate for hightemperature $S_{\text {config. }}$ of $\beta-\mathrm{B}_{106}$ is $\sim 0.5 R$ that is consistent with high-temperature instability of $\alpha-B_{12}$. According to Monte-Carlo simulations (see Ref.1 and references therein), the low-temperature $S_{\text {config. }}$ should be $\sim 5$ times smaller than that at high temperatures. Thus, at low temperatures the $\alpha \leftrightarrows \beta$ equilibrium line should have the slope $(\mathrm{d} p / \mathrm{d} T)_{\alpha-\beta} \approx\left(0.1 R \mathrm{~J} \mathrm{~mol}^{-1} \mathrm{~K}^{-1}\right) /\left(0.153 \mathrm{~cm}^{3}\right)^{1,8,59}$. As for the $(\mathrm{d} p / \mathrm{d} T)_{\alpha-\gamma}$, it tends to 0 when $\mathrm{T} \rightarrow 0$; and at very low temperatures the $\alpha \leftrightarrows \gamma$ equilibrium line should be parallel to the temperature axis. The low-temperature part of the diagram thus constructed seems to be the most thermodynamically consistent with all available experimental data and $a b$ initio calculations, and is very close to that previously reported by Oganov et $\mathrm{al}^{10}$.

Melting curve of $\boldsymbol{\beta}-\mathbf{B}_{\mathbf{1 0 6}}$. The experimental points on the $\beta-\mathrm{B}_{106}$ melting, both our experimental data and the previously reported values $^{43,44,60,61}$ are presented in Fig. 3. The disappearance of the diffraction lines of crystalline boron is accompanied by appearance of a broad halo typical for a liquid phase unambiguously points to the melting (Fig. 1a). Even slight decrease of the melt temperature leads to crystallization of $\beta-B_{106}$, indicative of equilibrium melting point and not metastable melting. At $5.45 \mathrm{GPa}$ the melting temperature was found to be $2440 \mathrm{~K}$. This is an intermediate value between the reported melting temperature of $2350-2370 \mathrm{~K}^{60,61}$ at ambient pressure and $2480 \mathrm{~K}$ at $7.7 \mathrm{GPa}^{43}$. The experiments at pressures below $2.5 \mathrm{GPa}$ have always led to blowouts and have not allowed establishing the low-pressure part of the melting curve.

According to Ref.62, $\Delta H_{\mathrm{L} / \beta}=50.2 \mathrm{~kJ} / \mathrm{mol}$ at ambient pressure. For our calculations, the melting temperature of boron at ambient pressure has been chosen as $2360 \mathrm{~K}$ (the mean value of $2350 \mathrm{~K}^{60}$ and $2370 \mathrm{~K}^{61}$ ). Since $\Delta V_{\mathrm{L} / \beta}$ is the value that strongly influences the slope of the melting curve, we have used it as a fitted parameter. The zeropressure atomic volume of $\beta$-boron has been calculated by extrapolation of the data on thermal expansion ${ }^{63}$. A good fit has been obtained with $\Delta \mathrm{V}_{\mathrm{L} / \beta}$ of $6.7 \%$ of the molar volume of crystalline boron at meting temperature which is close to the $\sim 5-10 \%$ volume change 

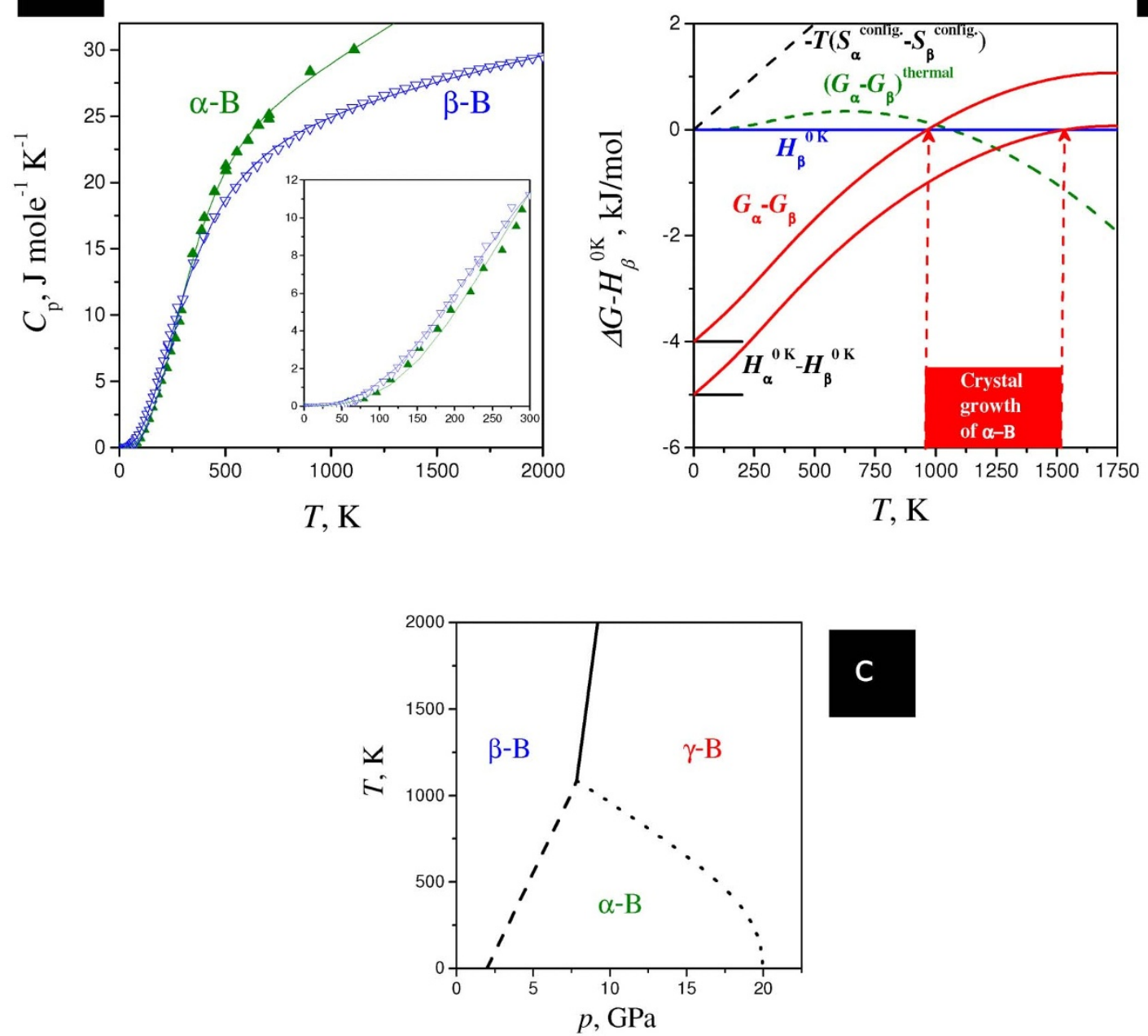

Figure $2 \mid$ (a) Heat capacity of $\alpha-B_{12}$ and $\beta-B_{106}$ at ambient pressure. The triangles show the experimental data ${ }^{56-58}$, while solid lines represent the data fit to the Holzapfel equation ${ }^{54,55}$. (b) Estimate for the formation enthalpy $\left(H_{\alpha}{ }^{0 \mathrm{~K}}-H_{\beta}{ }^{0 \mathrm{~K}}\right)$ of $\alpha-\mathrm{B}_{12}$ at ambient pressure $\left(\beta-\mathrm{B}_{106}\right.$ was considered as a standard state). The black dashed line shows the difference between Gibbs energies of $\alpha-B_{12}$ and $\beta-B_{106}$ due to the non-zero configurational entropy of $\beta$-phase, i.e. $T S_{\beta}{ }^{0 \mathrm{~K}}\left(S_{\alpha}{ }^{\mathrm{OK}}=0, S_{\beta}{ }^{0 \mathrm{~K}} \sim 0.5 R\right)^{49}$. The green dashed line represents the difference between thermal contributions (due to the difference in heat capacities) into the Gibbs energy of $\alpha-B_{12}$ and $\beta-B_{106}$. Blue solid line indicates the zero energy level chosen as $H_{\beta}{ }^{0 \mathrm{~K}}$. The highest and lowest temperatures of the synthesis of well-distinguishable $\alpha-B_{12}$ crystals allow one to suggest that $T_{\alpha-\beta}$ should be between $933 \mathrm{~K}^{50}$ and $1500 \mathrm{~K}^{51}$ (red rectangle area). Two red curves show the corresponding Gibbs energies of $\alpha-\mathrm{B}_{12}\left(G_{\alpha}-G_{\beta}\right)$ for two different values of $\left(H_{\alpha}{ }^{\mathrm{oK}}-H_{\beta}{ }^{\mathrm{oK}}\right)$; while these enthalpy values give the temperature limits $\left(933 \mathrm{~K}^{50}\right.$ and $\left.1500 \mathrm{~K}^{51}\right)$ for the $\alpha \leftrightarrows \beta$ equilibrium $\left(G_{\alpha}(\mathrm{T})-G_{\beta}(\mathrm{T})=0\right)$. The mean value of $\left(H_{\alpha}{ }^{0 \mathrm{~K}}-H_{\beta}{ }^{0 \mathrm{~K}}\right)$ is therefore about $-4.5 \mathrm{~kJ} / \mathrm{mol}$ if crystallization of $\alpha=B_{12}$ occurs at equilibrium conditions between these two temperatures. (c) Tentative low-temperature part of the boron phase diagram. The $\alpha \leftrightarrows \beta$ equilibrium line (dashed) crosses the pressure axis, similar to previous simulations ${ }^{1,10}$. The slope $(\mathrm{d} p / \mathrm{d} T)_{\alpha-\beta}=\Delta S_{\alpha-\beta} / \Delta V_{\alpha-\beta}$ is positive and has a non-zero value at $0 \mathrm{~K}$ due to the configurational enthropy of $\beta-\mathrm{B}_{106}$. The $(\mathrm{d} p / \mathrm{d} T)_{\alpha-\gamma} \rightarrow 0$ when $\mathrm{T} \rightarrow 0$; and at low temperatures the $\alpha \leftrightarrows \gamma$ equilibrium line (doted) is parallel to the temperature axis, while at higher temperature it joins the triple point defined by the intersection of the calculated $\alpha \leftrightarrows \beta$ (dashed) and experimental $\beta \leftrightarrows \gamma$ (solid) equilibrium lines.

reported in Refs.61, 64, 65 (Tab. 2) and is noticeably lower than the $20 \%$ value taken for the calculation of the melting curve in Ref.42. Thus, the experimental melting curve of $\beta$-boron up to 8 GPa may be described by the linear equation

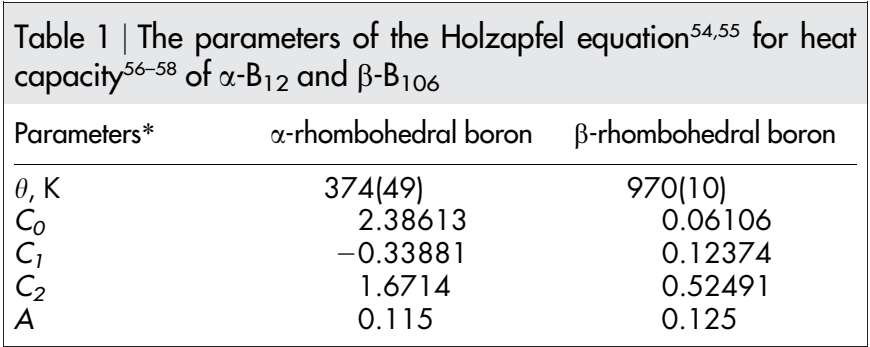

$$
T_{\mathrm{L} / \beta}=2360+15.5 p
$$

Equilibrium line between $\boldsymbol{\beta}-\mathrm{B}_{106}$ and $\boldsymbol{\gamma}-\mathrm{B}_{28}$. Our experiments at pressures above $10 \mathrm{GPa}$ have shown that samples quenched from $1600-2000 \mathrm{~K}$ contain only pure $\gamma-\mathrm{B}_{28}$, while the samples quenched from $8 \mathrm{GPa}$ contain only $\beta-\mathrm{B}_{106}$. These results well agree with experimental data of other groups ${ }^{13,50}$, and the $\beta \leftrightarrows \gamma$ equilibrium line that fits both our and literature data can be described by equation

$$
T_{\gamma / \beta}=-4534+707.8 p
$$

Equilibrium line between $\boldsymbol{\gamma}-\mathbf{B}_{\mathbf{2 8}}$ and $\mathbf{t}^{\prime}-\mathbf{B}_{\mathbf{5 2}}$. Above $10 \mathrm{GPa}$ the temperature seems to have more impact on the recovered boron 


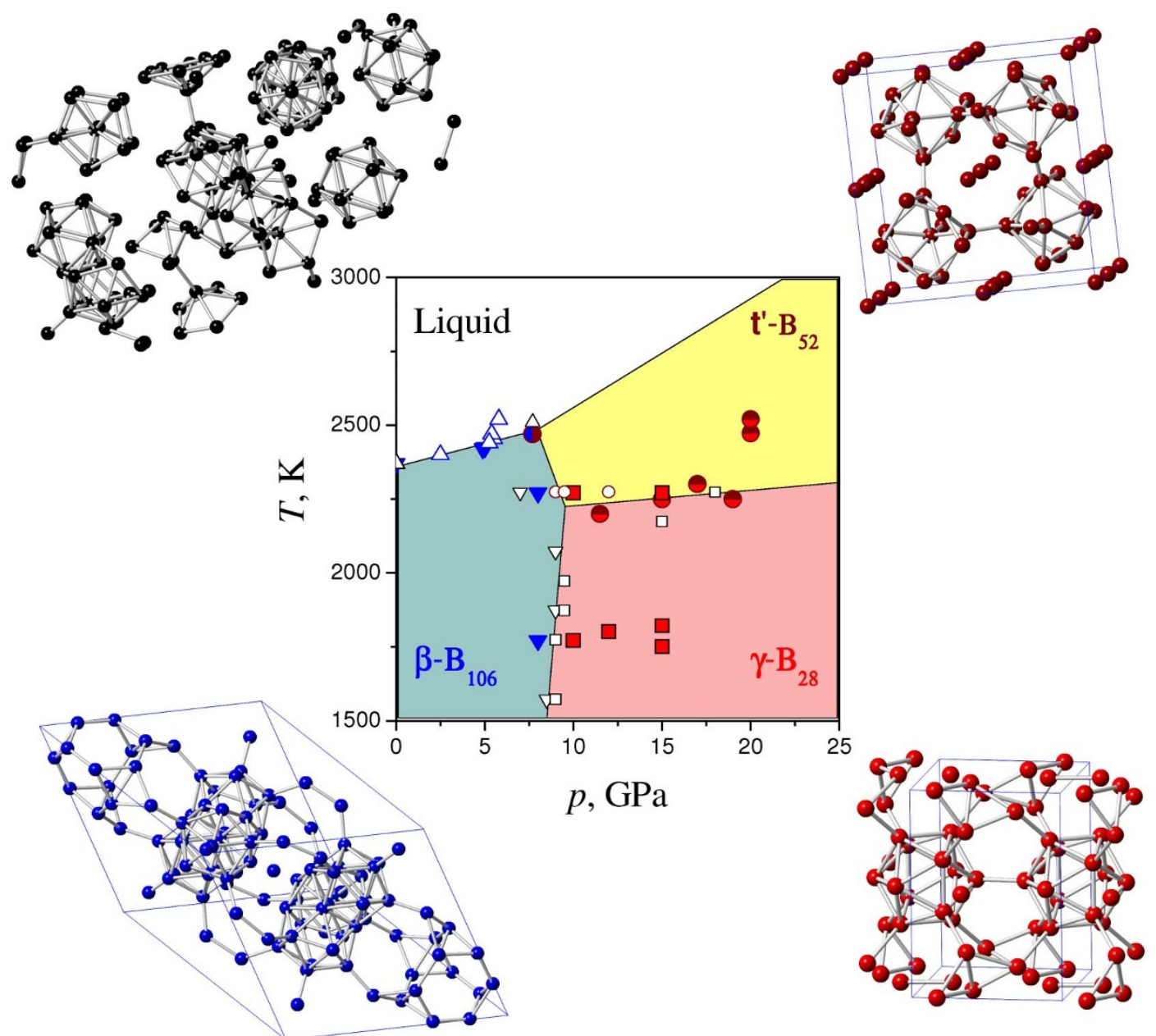

Figure $3 \mid$ Phase diagram of boron. The symbols show the experimental data. The solid lines represent the equilibria between different allotropes obtained by fitting the experimental points, as well as the unique melting curve of $\mathrm{t}^{\prime}-\mathrm{B}_{52}$ thermodynamically consistent with other phase equilibria. Down and up triangles show solid and melted $\beta-B_{106}$. Squares and circles correspond to $\gamma-B_{28}$ and $t^{\prime}-B_{52}$, respectively, recovered after quenching down to normal conditions. Small open symbols show literature data ${ }^{13}$. Color guide for symbols and structures: blue $-\beta-B_{106}{ }^{9}$, red $-\gamma-B_{28}{ }^{10}$, wine $-t^{\prime}-B_{52}{ }^{46}$, black - liquid $B$ (structural units of amorphous boron ${ }^{75}$ ).

Table 2 | Thermodynamic data on phase transformations in boron at pressures up to $25 \mathrm{GPa}$ and temperatures between 1500 and $3000 \mathrm{~K}$ $\begin{array}{lll}\Delta H_{Y / X}(\mathrm{~kJ} / \mathrm{mol}), \Delta S_{Y / X}(\mathrm{~kJ} / \mathrm{mol} \mathrm{K}) & \text { Fitting option } & \text { Experimental values and/or ab initio predictions } \\ \mathrm{Y} / \mathrm{X} & \text { or } \Delta V_{Y / X}\left(\mathrm{~cm}^{3} / \mathrm{mol}\right) & \text {. }\end{array}$

\begin{tabular}{|c|c|c|c|}
\hline$L / \beta$ & $\begin{array}{l}\Delta H=50 \\
\Delta V=15.5 \Delta H / 2360.0=0.32 \\
\Delta S=\Delta H / 2360.0=2.1 \cdot 10^{-2}\end{array}$ & $\begin{array}{l}\text { Fixed to experimental value } \\
\text { Constrained to experimental melting } \\
\text { curve and experimental } \Delta H_{L / \beta}\end{array}$ & $\begin{array}{l}\Delta H_{\text {melt }}=50.2^{43,62}, 48.93^{70} \\
V_{\text {melt }}=4.98^{64,65} \text { to } 5.22^{61} ; V_{\beta}=4.76^{63}\left(V_{300 \mathrm{~K}}=4.57^{59}\right) \\
\Delta V=0.22 \text { to } 0.46\end{array}$ \\
\hline$\gamma / \beta$ & $\begin{array}{l}\Delta H=2.5 \\
\Delta V=-707.8 \Delta H / 4534.0=-0.35 \\
\Delta S=-\Delta H / 4534.0=-5.5 \cdot 10^{-4}\end{array}$ & $\begin{array}{l}\text { Fixed to ab initio prediction } \\
\text { Constrained to experimental } \\
\text { transformation curve and } \Delta H_{\gamma / \beta}\end{array}$ & $\begin{array}{l}\Delta H_{\mathrm{OK}}=2.5^{10} \\
\Delta V_{300 \mathrm{~K},} 1 \mathrm{MPa}=4.25^{10}-4.57^{59}=-0.32\end{array}$ \\
\hline$t^{\prime} / \beta$ & $\begin{array}{l}\Delta H=8.8 \\
\Delta V=-160.2 \Delta H / 3755.7=-0.38 \\
\Delta S=\Delta H / 3755.7=2.3 \cdot 10^{-3}\end{array}$ & $\begin{array}{l}\text { Fitted } \\
\text { Constrained to experimental } \\
\quad \text { transformation curve and fitted } \Delta H_{t^{\prime} / \beta}\end{array}$ & $\Delta V_{300 K,} 1 \mathrm{MPa}=4.27^{46}-4.57^{59}=-0.30$ \\
\hline$t^{\prime} / \gamma$ & $\begin{array}{l}\Delta H=\Delta H_{r^{\prime} / \beta}-\Delta H_{y / \beta}=6.3 \\
\Delta V=5.1 \Delta H / 2176.0=0.01 \\
\Delta S=\Delta H / 2176.0=2.9 \cdot 10^{-3}\end{array}$ & $\begin{array}{l}\text { Adjusted to fitted } \Delta H_{\mathrm{t}^{\prime} / \beta} \text { and } \Delta H_{\gamma / \beta} \\
\text { Constrained to experimental } \\
\text { transformation curve and } \\
\text { adjusted } \Delta H_{\mathrm{t}^{\prime} / \gamma}\end{array}$ & $\Delta V_{300 K, 1} \mathrm{MPa}=4.27^{46}-4.25^{10}=0.02$ \\
\hline $\bar{L} / \mathrm{t}^{\prime}$ & $\begin{array}{l}\Delta H=\Delta H_{\mathrm{L} / \beta}-\Delta H_{\mathrm{t}^{\prime} / \beta}=41.2 \\
\Delta V=\Delta V_{\mathrm{L} / \beta}-\Delta V_{\mathrm{t}^{\prime} / \beta}=0.7 \\
\Delta S=\Delta S_{\mathrm{L} / \beta}-\Delta S_{\mathrm{t}^{\prime} / \beta}=1.9 \cdot 10^{-2}\end{array}$ & Defined by $\Delta G_{\mathrm{I}^{\prime} / \beta}$ and $\Delta G_{\mathrm{L} / \beta}$ & \\
\hline
\end{tabular}


allotrope as compared to the pressure. At 15-20 GPa, the samples quenched from $2500 \mathrm{~K}$ contained only mixture of $\gamma-\mathrm{B}_{28}$ and pseudocubic $\mathrm{t}^{\prime}-\mathrm{B}_{52}{ }^{46}$, while below $2200 \mathrm{~K} \mathrm{t}-\mathrm{B}_{52}$ (as well as $\alpha-\mathrm{B}_{12}$ ) could be observed as intermediate phase prior to crystallization of $\gamma-B_{28}$ in the stability domain of the latter ${ }^{12,13}$. At such high temperatures the kinetic factors do not play a decisive role anymore due to the intense diffusion. Thus, above $10 \mathrm{GPa}$ pseudo-cubic $\mathrm{t}^{\prime}-\mathrm{B}_{52}$ is stable at high temperatures, while $\gamma-B_{28}-$ at low and moderate temperatures.

All these results allowed us to define the domain of thermodynamic stability of $\gamma-B_{28}$ in the phase diagram (Fig. 3). The triple point between $\beta-B_{106}, \gamma-B_{28}$ and $t^{\prime}-B_{52}$ should be located at $\sim 9.6 \mathrm{GPa}$ and $\sim 2230 \mathrm{~K}$, while the equilibrium line between $\gamma-B_{28}$ and $t^{\prime}-B_{52}$ is described by the equation

$$
T_{\mathrm{t}^{\prime} / \gamma}=2176+5.1 p
$$

Only dense $\mathrm{t}^{\prime}-\mathrm{B}_{52}$ phase can explain such a low pressure slope of the $\mathrm{t}^{\prime} \leftrightarrows \gamma$ equilibrium line, contrary to other known tetragonal phases, $\mathrm{t}-\mathrm{B}_{52}$ and $\mathrm{t}-\mathrm{B}_{192}{ }^{46}$.

Equilibrium line between $\boldsymbol{\beta}-\mathbf{B}_{\mathbf{1 0 6}}$ and $\mathbf{t}^{\prime}-\mathbf{B}_{\mathbf{5 2}}$. The position of the $\beta-\gamma$-t' triple point, experimental data on phase stability of $\beta-B_{106}$ and the lowest pressure of $t^{\prime}-B_{28}$ formation (in some experiments this phase has been recovered at $\sim 7.7 \mathrm{GPa}$ ) allow one to draw the $\beta$ $\leftrightarrows \mathrm{t}^{\prime}$ equilibrium line (Fig. 3 ) which has negative pressure slope and follows the equation

$$
T_{\mathrm{t}^{\prime} / \beta}=3756-160.2 p
$$

This equilibrium line intersects the $\beta-\mathrm{B}_{106}$ melting curve at $\sim 8.0 \mathrm{GPa}$ and $\sim 2490 \mathrm{~K}$ that is the triple point between $\beta-\mathrm{B}_{106}$, $\mathrm{t}^{\prime}-\mathrm{B}_{52}$ and liquid.

\section{Discussion}

Among all boron allotropes mentioned above, the experimental thermodynamic values are known only for $\alpha-B_{12}$ and $\beta-B_{106}$, at least at ambient pressure. That is why it seems reasonable to use $\beta-B_{106}$ as standard state with known thermodynamic potentials, while for $\mathrm{L}, \mathrm{t}$ ' and $\gamma$ phases (X or $\mathrm{Y}$ ) the corresponding Gibbs energy should be corrected by a value of $\Delta G_{\mathrm{X} / \beta}$ defined by three parameters, i.e. $\Delta H_{\mathrm{X} / \beta}$, $\Delta V_{\mathrm{X} / \beta}$ and $\Delta S_{\mathrm{X} / \beta}$, independent of pressure and temperature in the first approximation. These parameters, if not known from experiment, can be obtained by fitting the experimental $p-T$ data to theoretical isopotential $\mathrm{X} \leftrightarrows \mathrm{Y}$ equilibrium lines (i.e. $\Delta G_{\mathrm{X} / \mathrm{Y}}(p, T)=$ $\left.\Delta G_{\mathrm{X} / \beta}(p, T)-\Delta G_{\mathrm{Y} / \beta}(p, T)=0\right)$.

Since the majority of experimentally observed equilibria between boron allotropes can be presented by straight lines, we did not complicated our analysis with exact formulae containing integrals. For example, $p \cdot \Delta V_{\mathrm{X} / \beta}$ and $\int \Delta V_{\mathrm{X} / \beta} \mathrm{d} p$ differ only by $\sim 5 \%$ (particularly, due to the close compressibilities of all boron allotropes $)^{66-69}$. Thus, the fitted parameters should be interpreted as average values over $p-T$ domain of experimental data.

In order to describe the high-temperature part of the boron phase diagram, one should know at least 9 parameters e.g. 3 expressions for $\Delta G_{\mathrm{X} / \beta}$ each containing 3 parameters, i.e. $\Delta H_{\mathrm{X} / \beta}, \Delta V_{\mathrm{X} / \beta}$ and $\Delta S_{\mathrm{X} / \beta}$. These dependences follow the equation

$$
\Delta G_{\mathrm{X} / \beta}=\Delta H_{\mathrm{X} / \beta}+p \Delta V_{\mathrm{X} / \beta}-T \Delta S_{\mathrm{X} / \beta} .
$$

For example, if one have such parameters for $\mathrm{X}=\mathrm{L}, \mathrm{t}$ ' and $\gamma$, two other Gibbs energies $\Delta G_{\mathrm{t}^{\prime} / \gamma}$ and $\Delta G_{\mathrm{L} / \mathrm{t}^{\prime}}$ will be related to them by simple general relationship

$$
\Delta G_{\mathrm{X} / \mathrm{Y}}=\Delta G_{\mathrm{X} / \beta}-\Delta G_{\mathrm{Y} / \beta}
$$

and will give us thermodynamically consistent equilibrium lines $\left(\Delta G_{\mathrm{X} / \beta}(p, T)=0\right)$. In order to establish these 9 parameters, one needs at least 9 independent experimental values characterizing those equilibria. Each experimental equilibrium line gives us two parameters: $\Delta H / \Delta S$ and $\Delta V / \Delta S$ according to the equation

$$
T_{\text {eq }}=\Delta H / \Delta S+p \Delta V / \Delta S
$$

that is a good approximation at high temperatures.

Four experimental equilibrium lines described above, i.e. $\mathrm{L} \leftrightarrows \beta$, $\beta \leftrightarrows \gamma, \mathrm{t}^{\prime} \leftrightarrows \beta$ and $\mathrm{t}^{\prime} \leftrightarrows \gamma$ give us 8 independent parameters. The ninth one we have chosen as experimental value of enthalpy of boron melting $\Delta H_{\mathrm{L} / \beta}$ (see Tab. 2) quite reproducible in independent experiments at ambient pressure ${ }^{43,62,70}$.

The fitted and fixed values of the thermodynamic parameters are given in Tab. 2 (data from Refs.10, 43, 46, 49, 59, 61-65, 70). One can observe a reasonable agreement of the fitted parameters with estimates for $\Delta V_{\mathrm{L} / \beta}$ and $\Delta V_{\mathrm{t}^{\prime} / \beta}$ based on volume measurements at high temperatures, as well as with $a b$ initio predicted $\Delta H_{\gamma / \beta}$ value at $0 \mathrm{~K}$. All this additionally indicates self-consistency and uniqueness of thermodynamic equations used, as well as real physical meaning of the fitted parameters.

The thermodynamic parameters of all mentioned above equilibria give the unique melting line of $\mathrm{t}^{\prime}-\mathrm{B}_{52}$, i.e.

$$
T_{\mathrm{L} / \mathrm{t}^{\prime}}=2186+37.4 p
$$

The parameters that define corresponding Gibbs energy are given in Tab. 2.

Figure 3 shows the phase diagram that represents all equilibria described above. At low pressures, two rhombohedral phases are stable, $\alpha-B_{12}$ and $\beta-B_{106}$. These allotropes have related structures ${ }^{4}$, rhombohedrally distorted fcc packing, produced by individual $\mathrm{B}_{12}$ icosahedra at low temperatures, and more complicated clusters at high temperatures. At pressures above $10 \mathrm{GPa}$, two other phases become stable, namely, $\gamma-B_{28}$ and $t^{\prime}-B_{52}$. Both of them have structures similar to distorted $\mathrm{NaCl}$ (orthorhombic and tetragonal distortions, respectively) ${ }^{10,46}$. It should also be noted that the common feature of two high-temperature phases $\left(\beta-B_{106}\right.$ and $\left.t^{\prime}-B_{52}\right)$ is a certain degree of intrinsic structural disorder (partially occupied Wickoff positions), which gives to them non-zero configurational entropy already at $0 \mathrm{~K}$ and, subsequently, results in their stability at high temperatures.

Finally, the equilibrium phase diagram of boron has been constructed at pressures up to $20 \mathrm{GPa}$ and temperatures up to $2500 \mathrm{~K}$ (Fig. 3). It has been experimentally proved that at least four boron phases, i.e. $\beta-B_{106}, \gamma-B_{28}, t^{\prime}-B_{52}$ and liquid boron, have $p-T$ domains of thermodynamic stability. Two triple points have been established, i.e. the first between $\beta-B_{106}, t^{\prime}-B_{52}$ and liquid at $\sim 8.0 \mathrm{GPa}$ and $\sim 2490 \mathrm{~K}$; and the second between $\beta-B_{106}, \gamma-B_{28}$ and $t^{\prime}-B_{52}$ at $\sim 9.6 \mathrm{GPa}$ and $\sim 2230 \mathrm{~K}$. Thermodynamic analysis leads to the unique melting curve of $\mathrm{t}^{\prime}-\mathrm{B}_{52}$ that is consistent with other experimentally established phase equilibria.

\section{Methods}

Multianvil experiments. Quenching experiments to $20 \mathrm{GPa}$ were performed using 6-8 type large-volume multianvil systems with octahedral pressure assemblies at Laboratoire des Sciences des Procédés et des Matériaux (LSPM-CNRS) and Bayerisches Geoinstitut (BGI). The experimental details are described elsewhere ${ }^{71,72}$ Pressure and temperature have been either directly measured (thermocouples and $p$ - $T$ phase transitions of reference materials) or estimated from previously obtained $p$ - $T$ calibration curves; in all cases, the uncertainties were estimated to be about $1 \mathrm{GPa}$ and $50 \mathrm{~K}$, respectively. Samples were gradually compressed to the desired pressure at ambient temperature, and then the temperature was increased with a rate of about $300 \mathrm{~K} / \mathrm{min}$ up to the desired value. After heating for 5-10 $\mathrm{min}$, the samples were quenched by switching off the power and then slowly decompressed.

In situ X-ray diffraction. In situ experiments to $7 \mathrm{GPa}$ were carried out using multianvil X-ray system MAX80 at beamline F2.1, DORIS III (HASYLAB-DESY). The experimental setup has been described elsewhere ${ }^{47}$. Energy-dispersive X-ray diffraction data were collected on a Canberra solid state Ge-detector with fixed Bragg angle $\theta=4.555(3)$ using a white beam collimated down to $100 \times 100 \mu^{2}$.

The sample temperature up to $2200 \mathrm{~K}$ was measured by a W3\%Re-W25\%Re thermocouple. The correction for the pressure effect on the thermocouple emf was made using the data of $\mathrm{Li}$ et $\mathrm{al}^{73}$. Above $2200 \mathrm{~K}$ the power - temperature calibration curve was linearly extrapolated to the high-temperature region (up to $2600 \mathrm{~K}$ ). 
Pressures at different temperatures were found from the $p-V-T$ equation of state of highly ordered $\left(P_{3}=0.98 \pm 0.02\right)$ graphite-like hexagonal boron nitride ${ }^{74}$.

The samples were gradually compressed to the required pressure at ambient temperature and then diffraction patterns were collected at the stepwise $(\sim 50 \mathrm{~K})$ temperature increase. With the storage ring operating at $4.44 \mathrm{GeV}$ and $150 \pm 50 \mathrm{~mA}$, diffraction patterns were collected for $1 \mathrm{~min}$ in real time. After heating, the samples were quenched by switching off the power, and then the pressure was slowly released down to ambient.

Ex situ X-ray diffraction. The recovered samples were studied by conventional powder X-ray diffraction using G3000 TEXT (Inel) diffractometer (Bragg-Brentano geometry) employing $\mathrm{CuK} \alpha 1$ radiation. The goniometer was aligned with high purity silicon $(a=5.431066 \AA)$ and the standard sample of $\operatorname{LaB}_{6}(a=4.15695 \AA)$. Synchrotron X-ray powder diffraction measurements $(\lambda=1.10347 \AA)$ have been performed at beamline I711, MAX II (MAX-lab); Debye-Scherrer geometry with rotating quartz capillary was used. Unit cell parameters, the size of the blocks of coherent scattering and strains were derived from the LeBail profile analysis performed using the GSAS program.

1. Ogitsu, T., Schwegler, E. \& Galli, G. $\beta$-Rhombohedral Boron: At the Crossroads of the Chemistry of Boron and the Physics of Frustration. Chem. Rev. 113, 3425-3449 (2013).

2. Oganov, A. R. \& Solozhenko, V. L. Boron: a hunt for superhard polymorphs. J. Superhard Mater. 31, 285-291 (2009)

3. Kurakevych, O. O. Superhard phases of simple substances and binary compounds of the B-C-N-O system: from diamond to the latest results (a Review). J. Superhard Mater. 31, 139-157 (2009).

4. Runow, P. Study of the $\alpha$ to $\beta$ transformation in boron. J. Mater. Sci. 7, 499-511 (1972).

5. Hoard, J. L., Hughes, R. E. \& Sands, D. E. The structure of tetragonal boron. J. Amer. Chem. Soc. 80, 4507-4515 (1958).

6. Wentorf, Jr, R. H. Boron: Another Form. Science 147, 49-50 (1965).

7. Ma, Y., Prewitt, C. T., Zou, G., Mao, H.-k. \& Hemley, R. J. High-pressure high temperature $\mathrm{x}$-ray diffraction of $\beta$-boron to $30 \mathrm{GPa}$. Phys. Rev. B 67, 174116 (2003).

8. Decker, B. F. \& Kasper, J. S. The crystal structure of a simple rhombohedral form boron. Acta Crystallogr. 12, 503-506 (1959).

9. Hughes, R. E. et al. Structure of beta-rhombohedral boron. J. Amer. Chem. Soc. $\mathbf{8 5}$ 361-362 (1963).

10. Oganov, A. R. et al. Ionic high-pressure form of elemental boron. Nature 457, 863-867 (2009).

11. Vlasse, M., Naslain, R., Kasper, J. S. \& Ploog, K. Crystal structure of tetragonal boron related to $\alpha-\mathrm{AlB}_{12}$. J. Solid State Chem. 28, 289-301 (1979).

12. Kurakevych, O. O., Le Godec, Y., Hammouda, T. \& Goujon, C. Comparison of solid-state crystallization of boron polymorphs at ambient and high pressures. High Pressure Res. 32, 30-38 (2012).

13. Qin, J. et al. Phase relations in boron at pressures up to $18 \mathrm{GPa}$ and temperatures up to $2200^{\circ}$ C. Phys. Rev. B 85, 014107 (2012).

14. Ekimov, E. A., Zibrov, I. P. \& Zoteev, A. V. Preparation of boron microcrystals via high-pressure, high-temperature pyrolysis of decaborane, $\mathrm{B}_{10} \mathrm{H}_{14}$. Inorg. Mater. 47, 1194-1198 (2011).

15. Oganov, A. R. \& Glass, C. W. Crystal structure prediction using evolutionary algorithms: principles and applications. J. Chem. Phys. 124, 244704 (2006).

16. Zhu, Q., Oganov, A. R., Glass, C. W. \& Stokes, H. T. Constrained evolutionary algorithm for structure prediction of molecular crystals: methodology and applications. Acta Crystallogr. B 68, 215-226 (2012).

17. Eremets, M. I., Struzhkin, V. W., Mao, H. K. \& Hemley, R. J. Superconductivity in boron. Science 293, 272-274 (2001).

18. Shimizu, K. et al. Superconductivity in $\alpha$-boron at Mbar pressure. Physica C 470, S631-S632 (2010).

19. Solozhenko, V. L., Le Godec, Y. \& Kurakevych, O. O. Solid-state synthesis of boron subnitride, $\mathrm{B}_{6} \mathrm{~N}$ : myth or reality? C. R. Chimie 9, 1472-1475 (2006).

20. Kurakevych, O. O. \& Solozhenko, V. L. Rhombohedral boron subnitride, $\mathrm{B}_{13} \mathrm{~N}_{2}$ by X-ray powder diffraction. Acta Crystallogr. C 63, i80-i82 (2007).

21. Adasch, V., Hess, K.-U., Ludwig, T., Vojteer, N. \& Hillebrecht, H. Synthesis, crystal growth and structure of $\mathrm{Mg}$ containing [beta]-rhombohedral boron: $\mathrm{MgB}_{17.4}$. J. Solid State Chem. 179, 2900-2907 (2006).

22. Solozhenko, V. L. \& Kurakevych, O. O. Chemical interaction in the B-BN system at high pressures and temperatures. Synthesis of novel boron subnitrides. J. Solid State Chem. 182, 1359-1364 (2009).

23. Kurakevych, O. O. \& Solozhenko, V. L. High-pressure route to superhard boronrich solids. High Press. Res. 31, 48-52 (2011).

24. Solozhenko, V. L., Kurakevych, O. O., Andrault, D., Le Godec, Y. \& Mezouar, M. Ultimate metastable solubility of boron in diamond: Synthesis of superhard diamond-like $\mathrm{BC}_{5}$. Phys. Rev. Lett. 102, 015506 (2009).

25. Nagatochi, T. et al. Superconductivity in Li-doped alpha-rhombohedral boron. Phys. Rev. B 83, art. 184507 (2011).

26. Hubert, H., Garvie, L. A. J., Buseck, P. R., Petuskey, W. T. \& McMillan, P. F. Highpressure, high-temperature syntheses in the B-C-N-O system. I. Preparation and Characterization. J. Solid State Chem. 133, 356-364 (1997).
27. Mukhanov, V. A., Sokolov, P. S. \& Solozhenko, V. L. On melting of $\mathrm{B}_{4} \mathrm{C}$ boron carbide under pressure. J. Superhard Mater. 34, 211-213 (2012).

28. Kurakevych, O. O. \& Solozhenko, V. L. Experimental study and critical review of structural, thermodynamic and mechanical properties of superhard refractory boron suboxide $\mathrm{B}_{6}$ O. J. Superhard Mater. 33, 421-428 (2011).

29. Solozhenko, V. L., Kurakevych, O. O., Turkevich, V. Z. \& Turkevich, D. V. Phase Diagram of the B-BN System at 5 GPa. J. Phys. Chem. B 114, 5819-5822 (2010).

30. Mukhanov, V. A., Kurakevych, O. O. \& Solozhenko, V. L. The interrelation between hardness and compressibility of substances and their structure and thermodynamic properties. J. Superhard Mater. 30, 368-378 (2008).

31. Mukhanov, V. A., Kurakevych, O. O. \& Solozhenko, V. L. Thermodynamic aspects of materials' hardness: prediction of novel superhard high-pressure phases. High Press. Res. 28, 531-537 (2008).

32. Mukhanov, V. A., Kurakevych, O. O. \& Solozhenko, V. L. Thermodynamic model of hardness: particular case of boron-rich solids. J. Superhard Mater. 32, 167-176 (2010).

33. McMillan, P. F. New materials from high-pressure experiments. Nat. Mater. 1, 19-25 (2002).

34. Nelmes, R. J. et al. Observation of inverted-molecular compression in boron carbide. Phys. Rev. Lett. 74, 2268 (1995).

35. Nieto-Sanz, D., Loubeyre, P. \& Mezouar, M. Equation of state and pressure induced amorphization of beta-boron from x-ray measurements up to $100 \mathrm{GPa}$. Phys. Rev. Lett. 89, 245501 (2002).

36. Solozhenko, V. L., Kurakevych, O. O. \& Kuznetsov, A. Y. Raman scattering from turbostratic graphite-like $\mathrm{BC}_{4}$ under pressure. J. Appl. Phys. 102, 063509 (2007).

37. Solozhenko, V. L. \& Kurakevych, O. O. Reversible pressure-induced structure changes in turbostratic BN-C solid solutions. Acta Crystallogr. B 61, 498-503 (2005).

38. Kurakevych, O. O., Solozhenko, E. G. \& Solozhenko, V. L. High-pressure study of graphite-like B-C phases. High Press. Res. 29, 605-611 (2009).

39. Solozhenko, V. L., Kurakevych, O. O., Solozhenko, E. G., Chen, J. \& Parise, J. B. Equation of state of graphite-like BC. Solid State Comm. 137, 268-271 (2006).

40. Haussermann, U., Simak, S. I., Ahuja, R. \& Johansson, B. Metal-nonmetal transition in the boron group elements. Phys. Rev. Lett. 90, 065701 1-4 (2003).

41. van Setten, M. J., Uijttewaal, M. A., de Wijs, G. A. \& de Groot, R. A. Thermodynamic stability of boron: The role of defects and zero point motion. J. Am. Chem. Soc. 129, 2458-2465 (2007).

42. Shirai, K., Masago, A. \& Katayama-Yoshida, H. High-pressure properties and phase diagram of boron. Phys. Stat. Solidi B 244, 303-308 (2007).

43. Brazhkin, V. V., Taniguichi, T., Akaishi, M. \& Popova, S. V. Fabrication of $\beta$ boron by chemical-reaction and melt-quenching methods at high pressures. J. Mater. Res. 19, 1643-1648 (2004).

44. Solozhenko, V. L., Kurakevych, O. O., Turkevich, V. Z. \& Turkevich, D. V. Phase diagram of the $\mathrm{B}-\mathrm{B}_{2} \mathrm{O}_{3}$ system at $5 \mathrm{GPa}$ : Experimental and theoretical studies. J. Phys. Chem. B 112, 6683-6687 (2008).

45. Horn, F. H. On the crystallization of simple rhombohedral boron from platinum. J. Electrochem. Soc. 106, 905-906 (1959).

46. Kurakevych, O. O. \& Solozhenko, V. L. Crystal structure of dense pseudo-cubic boron allotrope, $\mathrm{pc}-\mathrm{B}_{52}$, by powder X-ray diffraction. J. Superhard Mater. 35, 60-63 (2013)

47. Shimomura, O. Current activity of MAX80 at the Photon Factory. Physica B $+C$ 139-140, 292-300 (1986).

48. Glushko, V. P., Gurvich, L. V. \& Bergman, G. A. et al. (eds.) Thermodynamic Properties of Inorganic Materials. (Nauka, Moscow, 1981).

49. Masago, A., Shirai, K. \& Katayama-Yoshida, H. Crystal stability of $\alpha$ - and $\beta$-boron. Phys. Rev. B 73, 104102 1-10 (2006).

50. Parakhonskiy, G., Dubrovinskaia, N., Bykova, E., Wirth, R. \& Dubrovinsky, L. Experimental pressure-temperature phase diagram of boron: resolving the longstanding enigma. Sci. Rep. 1, art. 96 (2011).

51. Solov'ev, N. E., Makarov, V. S. \& Ugai, Y. A. The crystallization kinetics of amorphous boron. J. Less Comm. Met. 117, 21-27 (1986).

52. Kurakevych, O. O., Strobel, T. A., Kim, D. Y., Muramatsu, T. \& Struzhkin, V. V. $\mathrm{Na}$-Si clathrates are high-pressure phases: A melt-based route to control stoichiometry and properties. Cryst. Grow. Des. 13, 303-307 (2013).

53. Wong, J. \& Slack, G. A. Metals in beta-rhombohedral boron. Physica B + C 158, 627-628 (1989).

54. Holzapfel, W. B. Equations of state for solids under strong compression. High Press. Res. 16, 81-126 (1998).

55. Holzapfel, W. B. Approximate equations of state for solids from limited data sets. J. Phys. Chem. Solids 55, 711-719 (1994).

56. Gurvich, L. V., Khachkuruzov, E. A. \& Medvedev, V. A. Thermodynamic properties of individual substances. (eds. Glushko, V. P. \& al, e.) (Press of AN SSSR, Moscow, 1962).

57. Johnston, H. C., Hersh, H. N. \& Kerr, F. C. Low temperature heat capacities of inorganic solids. IV. The heat capacity of pure elementary boron in both amorphous and crystalline conditions between 13 and $305^{\circ} \mathrm{K}$. Some free energies of formation. J. Am. Chem. Soc. 73, 1112-1117 (1951).

58. Tsagareishvili, D. S. et al. Thermodynamic properties of $\alpha$-rhombohedral boron from 16.05 to 714.5 K. J. Less-Comm. Met. 117, 143-151 (1986).

59. Hoard, J. L., Sullenger, D. B., Kennard, C. H. L. \& Hughes, R. E. The structure analysis of $\beta$-rhombohedral boron. J. Solid State Chem. 1, 268-277 (1970). 
60. Kimpel, R. F. \& Moss, R. G. Melting point of 98.9 to $99.6 \%$ pure boron. J. Chem. Eng. Data 13, 231-234 (1968).

61. Krishnan, S., Nordine, P. C., Weber, J. K. R. \& Schiffman, R. A. Optical-properties and melting-point of pure boron. High Temp. Sci. 31, 45-57 (1991).

62. Stout, N. D., Mar, R. W. \& Boo, W. O. High-temperature enthalpy and enthalpy of fusion of boron by drop calorimetry. High Temp. Sci. 5, 241 (1973).

63. Lundstrom, T., Lonnberg, B. \& Bauer, J. Thermal expansion of betarhombohedral boron. J. Alloy. Comp. 267, 54-58 (1998).

64. Tsagareishvili, D. S. \& Tsagareishvili, G. V. Some thermoelastic properties of liquid boron at melting point. J. Less Comm. Met. 67, 541-550 (1979).

65. Tsagareishvili, G. V., Tsagareishvili, D. S. \& Khvedelidze, A. G. Estimation of some thermoelastic properties of [beta] rhombohedral boron in wide ranges of temperature and pressure. J. Less Comm. Met. 75, 141-145 (1980).

66. Sanz, D. N., Loubeyre, P. \& Mezouar, M. Equation of state and pressure induced amorphization of beta-boron from x-ray measurements up to $100 \mathrm{GPa}$. Phys. Rev. Lett. 89, 245501 (2002).

67. Le Godec, Y., Kurakevych, O. O., Munsch, P., Garbarino, G. \& Solozhenko, V. L. Equation of state of orthorhombic boron, $\gamma-\mathrm{B}_{28}$. Solid State Comm. 149 1356-1358 (2009)

68. Oganov, A. R., Solozhenko, V. L., Gatti, C., Kurakevych, O. O. \& Le Godec, Y. The High-Pressure Phase of Boron, gamma-B-28: Disputes and Conclusions of 5 Years after Discovery. J. Superhard Mater. 33, 363-379 (2011).

69. Le Godec, Y. Comparative Review of Theoretical and Experimental Equations of State of Orthorhombic Boron gamma-B-28. J. Superhard Mater. 33, 388-393 (2011).

70. Chase, M. W. J. NIST-JANAF Thermochemical Tables. 4th Edition (AIP Publishing, Melville, NY, 1998).

71. Rubie, D. C. Characterising the sample environment in multianvil high-pressure experiments. Phase Trans. 68, 431-451 (1999).

72. Solozhenko, V. L., Kurakevych, O. O. \& Le Godec, Y. Creation of nanostuctures by extreme conditions: High-pressure synthesis of ultrahard nanocrystalline cubic boron nitride. Adv. Mater. 24, 1540-1544 (2012).

73. Li, J., Hadidiacos, C., Mao, H.-K., Fei, Y. \& Hemley, R. J. Behavior of thermocouples under high pressure in a multi-anvil apparatus. High Press. Res. 23, 389-401 (2003).

74. Solozhenko, V. L. \& Peun, T. Compression and thermal expansion of hexagonal graphite-like boron nitride up to $7 \mathrm{GPa}$ and 1800 K. J. Phys. Chem. Solids 58, 1321-1323 (1997).
75. Kobayashi, M., Higashi, I. \& Takami, M. Fundamental structure of amorphous boron. J. Solid State Chem. 133, 211-214 (1997).

\section{Acknowledgments}

The authors thank Drs. C. Lathe and P.S. Sokolov for assistance in high-pressure experiments, Drs. T. Chauveau and C. Gundlach for help with X-ray diffraction studies and Profs. V.Z. Turkevich and A.R. Oganov for fruitful discussions. In situ experiments at HASYLAB-DESY have been carried out during beamtime allocated to Projects DESY-D-I-20090007 EC and DESY-D-I-20120445 EC and received funding from the European Community's Seventh Framework Programme (FP7/2007-2013) under grant agreement $n^{\circ}$ 226716. Multianvil experiments at the Bayerisches Geoinstitut were performed under the EU "Research Infrastructures: Transnational Access" Program (Contract No. 505320 (RITA) - High Pressure). Synchrotron X-ray powder diffraction studies at beamline I711, MAX-lab have been carried out during beamtime allocated to Proposal \# 20110330. This work was financially supported by the Agence Nationale de la Recherche (grant ANR-2011-BS08-018)

\section{Author contributions}

V.L.S. and O.O.K. equally participated in experimental work, data analysis and writing the manuscript.

\section{Additional information}

Competing financial interests: The authors declare no competing financial interests.

How to cite this article: Solozhenko, V.L. \& Kurakevych, O.O. Equilibrium $p$-T Phase Diagram of Boron: Experimental Study and Thermodynamic Analysis. Sci. Rep. 3, 2351; DOI:10.1038/srep02351 (2013)

(c) (i) $(-)$ This work is licensed under a Creative Commons Attribution-

Non Commercial-NoDerivs 3.0 Unported license. To view a copy of this license visit http://creativecommons.org/licenses/by-nc-nd/3.0 